\title{
The association of six-minutes walking test (6MWT) with cardiovascular disease risk among older women with type 2 diabetes mellitus in a rural primary health care: A pilot observational study
}

\author{
Rakhmat Ari Wibowo, ${ }^{1}$ Arum Tri Wahyuningsih, ${ }^{2}$ Rio Jati Kusuma, ${ }^{3}$ Wahyu Pamungkasih ${ }^{4}$ and \\ Denny Agustiningsih ${ }^{1 *}$ \\ 'Department of Physiology, Faculty of Medicine, Public Health, and Nursing, Universitas Gadjah Mada, Yogyakarta, \\ Indonesia \\ ${ }^{2}$ Department of Clinical Pathology and Laboratory Medicine, Faculty of Medicine, Public Health, and Nursing, Universitas \\ Gadjah Mada, Yogyakarta, Indonesia \\ ${ }^{3}$ Department of Health Nutrition, Faculty of Medicine, Public Health, and Nursing, Universitas Gadjah Mada, \\ Yogyakarta, Indonesia \\ ${ }^{4}$ Puskesmas Banguntapan II Bantul, Yogyakarta, Indonesia
}

ACCEPTED: 6 April 2020

\section{KEYWORDS \\ 6MWT \\ Cardiorespiratory fitness \\ Cardiovascular disease risk \\ Diabetes mellitus Older adults}

\begin{abstract}
Recent systematic review found that cardiovascular events contributed to approximately half of all deaths among patients with type 2 diabetes mellitus (T2DM). Several studies suggested that the six-minutes walking test (6MWT) could be a valuable prognostic tool for predicting cardiovascular disease (CVD) events in particular diseases. However, less is known concerning the role of 6MWT in predicting CVD events among patients with T2DM. Thus, this pilot observational study aimed to test the feasibility of conducting the 6MWT and to examine the association of measures collected during 6MWT with ASCVD risk estimator parameters for predicting CVD events among T2DM patients.Fourteen older women with T2DM in a rural primary health care were enrolled in this cross-sectional study. Blood pressure measurement, heart rate measurement, and blood sampling for HDL, LDL, and total cholesterol measurement were carried out during rest. Both heart rate and distance were measured at the end of the following $6 \mathrm{MWT}$. Feasibility data were collected.Recruitment rate and measurement completion rate were $85.7 \%$ and $40 \%$ respectively. No adverse events during the $6 \mathrm{MWT}$ were reported. Patient's heart rate at the end of 6MWT was correlated with diastolic blood pressure $(r=0.5 p=0.48)$. Multivariate analyses suggested that every one-meter increase in distance of $6 \mathrm{MWT}$, there is a decrease in diastolic blood pressure of $-0.9 \mathrm{mmHg}(p=0.01 ; 95 \% \mathrm{Cl}=$ -1.6 to -0.2 ). In conclusion, $6 \mathrm{MWT}$ is a feasible simple test which could provide a valuable prediction of ASCVD risk among older women with T2DM. Thus, this test should be considered to be conducted as a part of routine examination. Cohort study with a larger sample could be suggested to establish the usefulness of the 6MWT in predicting CVD risk.
\end{abstract}

(c) The Journal 2020. This article is distributed under a Creative Commons Attribution-ShareAlike 4.0 International license.

\section{Introduction}

Nowadays, researches put an increased focus on cardiovascular disease (CVD) complication in the management of T2DM. The risk of developing CVD increased with age. ${ }^{1}$ Among older adults, the prevalence of CVD in women is higher than in men due to the decline of estrogen and its receptor. ${ }^{2}$ In addition, women constitute a larger proportion

*Correspondence: denny.agustiningsih@gmail.com Department Physiology Faculty of Medicine, Public Health, and Nursing, Universitas Gadjah Mada, Jl. Farmako, Sekip Utara, Yogyakarta 55281, Indonesia of the elderly population in which the prevalence of CVD is greatest since they have a longer life expectancy than men. ${ }^{3} \mathrm{~A}$ recent systematic review found that approximately half of all deaths among patients with T2DM were attributed to CVD. ${ }^{4}$ Given the mortality burden of CVD among T2DM patients, comprehensive actions should be taken to provide a better prediction of CVD among T2DM patient than the traditional approach.

A scientific statement from the American Heart Association (2016) suggested that the addition of cardiorespiratory fitness (CRF) measurement to 
traditional risk scores significantly improves the prediction of CVD in the general population. ${ }^{5}$ Several mechanisms were postulated to explain the link between CRF and CVD, including improvement of insulin sensitivity, blood lipid, body composition, inflammation markers, vascular elasticity, and autonomic nervous system. Whilst the adding value of CRF measurement in the prediction of CVD among general populations is well identified, routine measurement of CRF is not readily available in both clinical and field settings because of the feasibility concerns, including cost, equipment, time, and expertise availability.

The six-minutes walking test (6MWT) is a practical test for estimating CRF, which is time-andcost-effective. Several studies suggested that the walking distance during the 6MWT could provide a valuable prediction of CVD among stable coronary heart disease patients or chronic obstructive pulmonary diseases. ${ }^{6,7}$ Despite its simplicity and its valuable prediction of CVD in particular diseases, the addition of heart rate measurement during 6MWT was also suggested by several authors to improve its accuracy. ${ }^{8}$ Also, the usefulness of 6MWT in the management of patients with T2DM is not wellstudied.

The aim of this pilot observational study is examining the recruitment rate of 6MWT, measurement completion rate and the association between the 6MWT and CVD risks among women with T2DM in a rural primary health care. Walking distance and heart rate response will be measured in the 6MWT. This study will utilise the ASCVD risk estimator plus tool since this tool has been widely used for predicting cardiovascular events among patients with T2DM.

\section{Method}

\subsection{Subject and design}

This is a cross-sectional study design to investigate the association between the walking distance and heart rate response during 6-MWT with CVD risk measured using ASCVD risk estimator plus tool among older women with T2DM. Patients were eligible if they were women 55 years or older, had
T2DM and registered in Program Pengelolaan Penyakit Kronis (Prolanis) at Puskesmas Banguntapan II, Bantul. Patients were excluded if they had physical disabilities which obstructed them to walk one block and had a history of T2DM complications. There were 51 patients ( 16 men, 35 women) registered in Prolanis who were eligible for this study. This study has been approved by the Faculty of Medicine, Public Health, and Nursing Research Ethics Committee (KE/0001/01/2020). A written informed consent was obtained from the participant.

\subsection{Study Protocol}

As a pilot study, the recruitment rate, measurement completion rate, and adverse event were collected. Recruitment rate was defined as the proportion of eligible patients who agreed to participate in the 6MWT. Measurement completion rate was defined as the proportion of recruited participants who were able to complete all measurements.

Asingle 6MWT without practicewasadministered according to a standard guideline. ${ }^{6}$ Patients were instructed to walk continuously on a premeasured field $33 \mathrm{~m}$ in length. Health professional who was administering the test provided encouragement every minute in a standardised fashion. Total distance walked in six minutes was recorded. Patients' heart rate before the test was recorded using finger pulse oximetry in sitting position. ${ }^{9}$ Patients' heart rate at the end of 6MWT were recorded in standing positions.

Patients' weight, body fat percentage, visceral fat, and muscle mass were measured using Omron BF508 bio-impedance analyser. ${ }^{10}$ Patients' height was measured using a height scale. Body mass index (BMI) was calculated based on patients' weight and height. Patients' blood sample was collected for HbA1c, fasting blood glucose, total cholesterol, triglyceride, and HDL-cholesterol. LDL-cholesterol was estimated using a Friedewald calculation. ${ }^{11}$ Blood pressure was measured using an Omron blood pressure monitor in a sitting position. ${ }^{12}$

\subsection{Statistical analysis}

We measured all variables as continuous data and descriptively presented them in mean and standard 
deviation. Participants were also grouped by hypertension category and t-test was conducted to examine the difference among them. We conducted a Pearson correlation test to examine the association between 6MWT measures with ASCVD risk estimator plus measures. Multiple linear regression tests were conducted to predict the value of ASCVD risk estimator plus parameters from the 6MWT results. The regression was adjusted from patients' BMI.

\section{Result}

\subsection{Feasibility measures}

We mailed 35 women patients an invitation to participate, and 30 responded a week later with interest resulting in a recruitment rate of 30/35 (85.7\%). Of those responding, 9 participants could not be reached for the 6MWT scheduling (6MWT completion rate of $70 \%), 7$ participants had missing laboratory and body composition measurement data (laboratory examination completion rate of $66.7 \%)$. In total, 14 patients were able to complete all measurements resulting in a measurement completion rate of $14 / 35$ (40\%). There were no adverse events resulting from the 6MWT.

\subsection{Outcome variables}

Patients in this study were 59 (7.5) years old on average. Their average BMI was $23.7(4.2) \mathrm{kg} / \mathrm{m}^{2}$. Patients' average systolic blood pressure, diastolic blood pressure, total cholesterol, HDL-cholesterol, and LDL cholesterol were 148 (25.3) $\mathrm{mmHg}, 77.2$ (11.7) mmHg, 186 (31.6) mg/dl, 56.9 (10.6) mg/dl, and 94.9 (23.1) mg/dl, respectively. Characteristics of the patients in this study were presented in Table 1. There was no difference on age, body composition, lipid profile and 6MWT results between normotensive and hypertensive patients $(p>0.05)$.

Pearson correlation test showed that only heart rate response at the end of 6MWT had a significant association with systolic blood pressure $(r=0.676$ $p=0.008)$ and diastolic blood pressure $(r=0.632$ $p=0.015)$ which were parameters measured for ASCVD risk estimator plus (Table 2). After adjusted with participants' BMI, multiple linear regression showed that walking distance had a probability of being associated with a difference in patients' diastolic blood pressure (Coefficient -0.93 95\% confidence interval -1.64 to -0.22 ) (Table 3 ). A more prominent predictive value of 6MWT was found among normotensive patients (Table 4). Both exercise heart

Table 1. Characteristics of participants

\begin{tabular}{lllllll}
\hline \multirow{2}{*}{ Variable } & \multicolumn{2}{l}{ All } & \multicolumn{2}{l}{ Hypertensive } & \multicolumn{2}{l}{ Normotensive } \\
\cline { 2 - 7 } & Mean & SD & Mean & SD & Mean & SD \\
\hline Age (years) & 59 & 7.5 & 59.8 & 9.7 & 58.0 & 3.3 \\
BFP (\%) & 34.8 & 3.9 & 36.3 & 3.4 & 32.7 & 4.0 \\
VF (point) & 8.2 & 4.6 & 9.9 & 4.9 & 6.0 & 3.3 \\
LM (\%) & 22.6 & 1.6 & 22.1 & 1.5 & 23.2 & 1.5 \\
BMI (kg/m²) & 23.7 & 4.2 & 25.1 & 4.5 & 22.4 & 3.6 \\
SBP (mmHg) & 148.1 & 25.3 & 164.9 & 17.5 & 125.7 & 13.8 \\
DBP (mmHg) & 77.2 & 11.7 & 83.6 & 8.6 & 68.7 & 9.9 \\
TC (mg/dl) & 186.1 & 31.7 & 181.6 & 25.3 & 192.0 & 40.4 \\
HDL-C (mg/dl) & 56.9 & 10.6 & 60.6 & 10.8 & 51.8 & 8.7 \\
LDL-C (mg/dl) & 94.9 & 23.1 & 91.8 & 19.1 & 98.9 & 28.9 \\
TG (mg/dl) & 166.1 & 48.4 & 146.1 & 26.8 & 192.7 & 60.0 \\
RHR (x/minute) & 85.2 & 17.0 & 86 & 21 & 84 & 11 \\
EHR (x/minute) & 104.9 & 16.9 & 111 & 17 & 97 & 14 \\
Exercise intensity (\%MaxHR) & 65.3 & 11.2 & 69.4 & 11.7 & 59.8 & 8.4 \\
WD (m) & 161.0 & 7.5 & 160.2 & 9.7 & 162 & 3.3 \\
\hline
\end{tabular}

BFP: body Fat Percentage; VF: Visceral Fat; LM: Lean Mass; BMI: Body Mass Index; SBP: Systolic Blood Pressure; DBP: Diastolic Blood Pressure; TC: Total Cholesterol; HDL-C: High Density Lipoprotein Cholesterol; LDL-C: Low Density Lipoprotein Cholesterol; TG:Triglyceride; RHR: Resting Heart Rate; HER: Exercise Heart Rate; WD: Walking Distance 
Table 2. Association between 6MWD and ASCVD risk estimator plus variables

\begin{tabular}{llllll}
\hline \multirow{2}{*}{ 6MWT } & \multicolumn{4}{l}{ ASCVD risk estimator plus } & \multicolumn{4}{l}{ (r coefficient) } & & HDL \\
\cline { 2 - 6 } & SBP & DBP & TC & LDL & 0.260 \\
\hline RHR & 0.482 & 0.506 & 0.249 & 0.204 & 0.292 \\
EHR & $0.676^{* *}$ & $0.632^{*}$ & 0.292 & -0.061 & $0 .-238$ \\
Walking Distance & -0.114 & 0.187 & 0.17 & 0.026 & \\
\hline
\end{tabular}

"Significant $(p<0.05) ;{ }^{* *}$ Significant $(p<0.01)$

SBP: Systolic Blood Pressure; DBP:Diastolic Blood Pressure; TC: Total Cholesterol; HDL-C: High Density Lipoprotein Cholesterol; LDL-C: Low Density Lipoprotein Cholesterol; RHR: Resting Heart Rate; HER: Exercise Heart Rate.

Table 3. Multiple linear regression among all participants

\begin{tabular}{llllll}
\hline \multirow{2}{*}{ 6MWT } & \multicolumn{4}{l}{ ASCVD risk estimator plus (Coefficient [95\% Confidence Interval]) } \\
\cline { 2 - 6 } & SBP & DBP & TC & LDL & HDL \\
\hline \multirow{2}{*}{ RHR } & $-0.71(-1.95$ to & $-0.38(-0.73$ to & $1.55(-0.78$ to & $-4.72(-45.38$ to & $0.07(-6.70$ to \\
& $0.53)$ & $-0.02)^{*}$ & $3.88)$ & $35.95)$ & $6.84)$ \\
EHR & $0.70(-0.56$ to & $0.14(-0.23$ to & $-0.43(-2.79$ to & $0.42(-13.21$ to & $-0.57(-2.83$ to \\
& $1.96)$ & $0.49)$ & $1.93)$ & $14.06)$ & $1.70)$ \\
Walking Distance & $-2.06(-4.51$ to & $-0.93(-1.64$ to & $1.37(-3.24$ to & $0.417(-2.29$ to & $0.17(-0.14$ to \\
\hline
\end{tabular}

${ }^{*}$ Significant $(p<0.05)$

SBP: Systolic Blood Pressure; DBP:Diastolic Blood Pressure; TC: Total Cholesterol; HDL-C: High Density Lipoprotein Cholesterol; LDL-C: Low Density Lipoprotein Cholesterol; RHR: Resting Heart Rate; HER: Exercise Heart Rate.

Table 4. Multiple linear regression among normotensive patients

\begin{tabular}{llllll}
\hline \multirow{2}{*}{ 6MWT } & \multicolumn{4}{l}{ ASCVD risk estimator plus (Coefficient [95\% Confidence Interval]) } \\
\cline { 2 - 6 } & SBP & DBP & TC & LDL & HDL \\
\hline \multirow{2}{*}{ RHR } & $-2.92(-52.71$ to & $-0.81(-1.12$ to & $7.33(-156.21$ to & $6.42(-51.67$ to & $-0.02(-14.26$ to \\
& $46.88)$ & $-0.51)^{*}$ & $100.89)$ & $64.51)$ & $14.22)$ \\
EHR & $1.01(-28.22$ to & $0.21(0.02$ to & $7.33(-68.60$ to & $-3.21(-37.31$ to & $0.75(-7.61$ to \\
& $30.24)$ & $0.39)^{*}$ & $83.26)$ & $30.89)$ & $9.11)$ \\
Walking Distance & $-4.34(-64.19$ to & $-0.94(-1.31$ to & $18.71(-72.54$ to & $10.87(-58.94$ to & $4.36(-12.76$ to \\
& $55.49)$ & $-0.57)^{*}$ & $109.96)$ & $80.68)$ & $21.47)$ \\
\hline
\end{tabular}

${ }^{*}$ Significant $(p<0.05)$

SBP: Systolic Blood Pressure; DBP:Diastolic Blood Pressure; TC: Total Cholesterol; HDL-C: High Density Lipoprotein Cholesterol; LDL-C: Low Density Lipoprotein Cholesterol; RHR: Resting Heart Rate; HER: Exercise Heart Rate.

rate and walking distance could predict patients' diastolic blood pressure with coefficient of 0.21 ( 0.02 to 0.39$)$ and $-0.94(-1.31$ to -0.57$)$ respectively.

\section{Discussion}

The primary aim of this pilot study is to investigate the feasibility of adding a 6MWT into routine measurement in a rural primary health care. The high recruitment rate $(85.7 \%)$ represented a high demand of the 6MWT among women patients with T2DM. Also, there were no adverse events. To be feasible, participation of subjects in the outcome measurements is important. Due to the administration of a 6MWT in an additional day from the routine visit, there was a high burden on participants resulting in a low measurement completion rate (40\%). Thus, future studies and practices should consider conducting the 6MWT in the same day with the routine visit to minimize burden on participants.

Our study also found association between 6MWT results and CVD risk factors. Pearson correlation test and multiple linear regression also suggested an association between 6MWT measures and blood pressure as a part of CVD risk factors. A strong linear correlation was found between heart rate response after 6MWT and blood pressure. This association is in agreement with the previous study. A cohort 
study of 10418 healthy men found that the risk of hypertension increased in men with higher exercise heart rate response. ${ }^{13}$

After adjustment for participants' BMI, heart rate still had an association with blood pressure. Participants' resting heart rate was inversely correlated with their diastolic blood pressure. Every 1 point increase in heart rate could result in a $0.38 \mathrm{mmHg}$ decrease in diastolic blood pressure. This finding contrast with several previous studies. Several cross-sectional with large samples found that resting heart rate positively correlated with blood pressure. ${ }^{14,15,16}$ The disagreement in the relationship between resting heart and blood pressure in this study could be attributed to a Hawthorne effect. Since participants' resting heart rate was recorded on a similar day with the 6MWT schedule, participants became aware that they would be examined. It could result in an autonomic nervous system response which could influence their resting heart rate.

Walking distance covered during 6MWT were inversely correlated with participants' diastolic blood pressure after adjustment for their BMI. Every one 1 meter increase of walking distance could result in a $0.93 \mathrm{mmHg}$ decrease in diastolic blood pressure. Walking distance in the 6MWT reflected the aerobic capacity of the participant. A previous study found that higher aerobic capacity could reduce the risk of arterial stiffness. ${ }^{17}$ Since arterial stiffness resulted in a greater influence on diastolic blood pressure than systolic blood pressure ${ }^{18}$, this mechanism could explain the inverse association between walking distance with participants' diastolic blood pressure.

Whilst the predictive value of 6MWT became prominent among normotensive patients, there was lack of association between 6MWT and traditional CVD risks among hypertensive patients in our study. Baroreceptor impairment among hypertensive patients could reduce the sensitivity to the 6MWT load. ${ }^{19}$ A submaximal aerobic test could result in a better sensitivity than 6MWT but the risk should be considered. The weak association of aerobic fitness test in predicting CVD risks among hypertensive patients is in agreement with a previous cohort study $^{20}$. Whilst normotensive patients with high aerobic fitness were associated with 2.27 times less risk of CVD mortality, hypertensive patients with high aerobic fitness were only associated with 1.1 times less risk of CVD mortality. ${ }^{20}$ Similar with that cohort study, the presence of pharmacotherapy among hypertensive patients could be the confounding factor between aerobic fitness and other CVD risks. Thus, considering for pharmacotherapy as a confounding factor could be suggested to future studies.

We also found lack of association between 6MWT result and blood lipid profile. However, previous prospective cohort found that patients with high fitness level were associated with lower risk of the incidence of dyslipidemia. ${ }^{20}$ Since blood lipid was also strongly associated with several factors that were not controlled in our study, such as diet and family history, these could be confounding factors that interfere between 6MWT and blood lipid.

This cross-sectional study also found that older women patients with T2DM in a rural primary health care also had hypertension as comorbidity. In average, their systolic blood pressure and diastolic blood pressure were $148(25.3) \mathrm{mmHg}$ and 77.2 (11.7) $\mathrm{mmHg}$ respectively. Whilst they did not have a good blood pressure control, they had a good control of blood lipid. Their average total cholesterol, HDLcholesterol, LDL-cholesterol, and triglyceride were in a normal range. Based on mean BMI, participants had healthy weight. However, their mean visceral fat was categorised as a high level. In average, the 6MWT resulted in $65.3 \%$ of participants' maximum heart rate. Thus, this test represents a moderate intensity activity that could be used for predicting cardiorespiratory fitness in T2DM patients.

The results from this study suggested an additional value of a simple fitness testing which could be associated with traditional CVD risks particularly in normotensive T2DM patients. However, the findings from this study should be applied with cautious. This study did not show a causal relationship. Longitudinal studies with a large sample and cardiovascular events-related outcome should be conducted to investigate the predictive value of 6MWT on CVD risk among T2DM patients. Several confounding factors such as pharmacotherapy, diet, and family history should also be considered. Statistical analysis for 
adjustment of the missing data was not conducted in this study. This could result in a high risk of bias. Thus, further study should consider missing data in statistical analysis.

\section{Conclusion}

This pilot study provided evidence that 6MWT is feasible to be conducted in a rural primary health care. Implementation of 6MWT among older women with T2DM could provide additional values for predicting CVD risk, but large longitudinal study with a rigorous method should be conducted in the future. Considering its additional values and the high demand of this test, stakeholders should consider to include this test into routine examination among older women with T2DM in primary health care.

\section{Competing interests}

This study was funded by Community Empowerment Grants from Faculty of Medicine, Public Health, and Nursing, Universitas Gadjah Mada. Authors do not have any financial, professional, or personal relationships that are relevant to the submitted work.

\section{References}

1. North BJ, Sinclair DA. The intersection between aging and cardiovascular disease. Circ. Res. 2012; 110:1097-1108

2. Garcia M, Mulvagh SL, Merz CN, Buring JE, Manson JE. Cardiovascular disease in women: clinical perspectives. Circ. Res. 2016; 118:12731293

3. World Health Organization. Global Health Observatory (GHO) data: Life expectancy. Geneva, Swiss. 2020

4. Einarson TR, Acs A, Ludwig C, Panton UH. Prevalence of cardiovascular disease in type 2 diabetes: a systematic literature review of scientific evidence from across the world in 2007-2017. Cardiovasc Diabetol. 2018;17:83.

5. Ross $R$, et al. Importance of assessing cardiorespiratory fitness in clinical practice: a case for fitness as a clinical vital sign: a scientific statement from the American Heart Association. Circulation. 2016;134(24):e653-e699.

6. ATS Committee on Proficiency Standards for
Clinical Pulmonary Function Laboratories. ATS statement: guidelines for the six-minute walk test. Am J Respir Crit Care Med. 2002; 166:111117.

7. Beatty AL, Schiller NB, Whooley MA. Six-minute walk test as a prognostic tool in stable coronary heart disease: data from the Heart and Soul Study. Arch Intern Med. 2012; 172:1096-1102.

8. Mänttäri $A$, Suni J, Sievänen $H$, et al. Six-minute walk test: a tool for predicting maximal aerobic power (vo2 max) in healthy adults. Clin Physiol Funct Imaging. 2018; 38(6): 1038-1045

9. Iyriboz Y, Powers S, Morrow J, Ayers D, Landry G. Accuracy of pulse oximeters in estimating heart rate at rest and during exercise. Br J Sports Med. 1991;25:162-4

10. Dehghan M, Merchant AT. Is bioelectrical impedance accurate for use in large epidemiological studies? Nutr J. 2008;7:26.

11. Razi F, Forouzanfar K, Bandarian F, Nasli-Esfahani E. LDL-cholesterol measurement in diabetic type 2 patients: a comparison between direct assay and popular equations. J Diabetes Metab Disord. 2017;16:43.

12. Chahine MN, Topouchian J, Zelveian $P$, et al. Validation of BP devices QardioArm ${ }^{\circledR}$ in the general population and Omron M6 Comfort $^{\circledR}$ in type II diabetic patients according to the European Society of Hypertension International Protocol (ESH-IP). Med Devices. 2017;11:11-20.

13. Prasad VK, Hand GA, Sui $X$, et al. Association of exercise heart rate response and incidence of hypertension in men. Mayo Clin Proc. 2014;89(8):1101-1107.

14. Aladin A, Al Rifai M, Rasool S, et al. The association of resting heart rate and incident hypertension: The Henry Ford Hospital Exercise Testing (FIT) project. American Journal of Hypertension. 2016; 29(2): 251-257.

15. Christofaro DGD, Casonatto J, Vanderlei LCM, Cucato GG, Dias RMR. Relationship between resting heart rate, blood pressure and pulse pressure in adolescents. Arq Bras Cardiol. 2017;108(5):405-410.

16. Liu L, Mizushima S, Ikeda K, Nara Y, Yamori Y. Resting heart rate in relation to blood pressure: results from the World Health Organization- 
Cardiovascular Disease and Alimentary Comparison study. Int J Cardiol. 2010;145:73-74.

17. Ferreira I, Twisk J, Stehouwer C, Van Mechelen, W, Kemper H. Longitudinal changes in \&vo2max: associations with carotid imt and arterial stiffness. Med Sci Sports Exerc. 2003; 35(10): 1670-1678.

18. Liu J, Sui X, Lavie CJ, Zhou H, Park YM, Cai B, et al. Effects of cardiorespiratory fitness on blood pressure trajectory with aging in a cohort of healthy men. J Am Coll Cardiol. 2014; 64:124553.

19. Buñag RD, Eriksson L, Krizsan D. Baroreceptor reflex impairment and mild hypertension in rats with dietary-induced obesity. Hypertension. 1990; 15(4): 397-406

20. Church TS, Kampert JB, Gibbons LW, Barlow CE, Blair SN. Usefulness of cardiorespiratory fitness as a predictor of all-cause and cardiovascular disease mortality in men with systemic hypertension. Am J Cardiol. 2001; 88(6):651-6.

21. Watanabe N, S Sawada S, Shimada K, et al. Relationship between cardiorespiratory fitness and non-high-density lipoprotein cholesterol: a cohort study. J Atheroscler Thromb. 2018; 25(12):1196-1205. 